Dementia

and Geriatric

Cognitive Disorders

Dement Geriatr Cogn Disord 2018;46:81-89

\title{
Dementia Patients Are More Sedentary and Less Physically Active than Age- and Sex-Matched Cognitively Healthy Older Adults
}

\author{
Yvonne A.W. Hartman ${ }^{a}$ Esther G.A. Karssemeijer ${ }^{\text {b, c }}$ \\ Lisanne A.M. van Diepen ${ }^{a}$ Marcel G.M. Olde Rikkert ${ }^{b, c}$ \\ Dick H.J. Thijssen ${ }^{a}$ d \\ aDepartment of Physiology, Radboud Institute for Health Sciences, Radboud University \\ Medical Center, Nijmegen, The Netherlands; ${ }^{b}$ Department of Geriatric Medicine, Donders \\ Institute for Brain, Cognition and Behavior, Radboud University Medical Center, Nijmegen, \\ The Netherlands; ${ }^{C}$ Radboudumc Alzheimer Center, Radboud University Medical Center, \\ Nijmegen, The Netherlands; ${ }^{d}$ Research Institute for Sport and Exercise Sciences, Liverpool \\ John Moores University, Liverpool, UK
}

\section{Keywords}

Sedentary behaviour - Dementia · Physical activity · Actigraphy

\begin{abstract}
Aims: The aim of this study was to examine physical activity and sedentary behaviour characteristics of ambulatory and community-dwelling patients with dementia compared to cognitively healthy age-, sex- and weight-matched controls. Methods: In this cross-sectional study, we included community-dwelling dementia patients ( $n=45$, age $79.6 \pm 5.9$ years, MiniMental State Examination [MMSE] $22.8 \pm 3.2$ ) and matched controls ( $n=49$, age $80.0 \pm 7.7$ years, MMSE $29.0 \pm 1.2$ ). Participants wore a wrist accelerometer for 7 days to assess sedentary time, sedentary bout duration and time spent in very light, light-to-moderate and moderateto-vigorous physical activities. Results: Relative sedentary time and sedentary bout duration was significantly higher in dementia patients than in controls (median [interquartile range] $57 \%$ [49-68] vs. 55\% [47-59] and 18.3 [16.4-21.1] $\mathrm{min}$ vs. 16.6 [15.3-18.4] $\mathrm{min}, p=0.042$ and $p=0.008$, respectively). In addition, dementia patients spent a lower percentage of their waking time in light-to-moderate and moderate-to-vigorous intensity physical activities (20\% [15-23] vs. 22\% [18-25] and 5\% [2-10] vs. $10 \%$ [5-13], $p=0.017$ and $p=0.001$, respectively). Conclusion: We revealed that dementia patients are more sedentary and perform less phys-
\end{abstract}

Y.A.W.H. and E.G.A.K. share first authorship and contributed equally to this article.

Prof. Dr. Dick H.J. Thijssen

Department of Physiology, Radboud University Medical Center

Philips van Leijdenlaan 15

NL-6525 EX Nijmegen (The Netherlands)

E-Mail dick.thijssen@ radboudumc.nl 
Hartman et al.: Dementia Patients Are More Sedentary and Less Physically Active than Age- and Sex-Matched Cognitively Healthy Older Adults

ical activity than cognitively healthy controls. This may have clinically important consequences, given the observation that sedentary behaviour and little physical activity independently predict all-cause mortality and morbidity.

(C) 2018 The Author(s)

Published by S. Karger AG, Basel

\section{Introduction}

The incidence and prevalence of dementia is rising [1]. Pharmacological treatment to slow disease progression shows limited benefits on cognitive functioning [2]. Therefore, nonpharmaceutical therapies are needed to attenuate or slow cognitive decline. Engagement in moderate-to-vigorous physical activity (i.e., exercise) is one of the most important modifiable risk factors for dementia [3].

Moreover, exercise interventions have beneficial effects on cognitive function in older adults with dementia [4]. Interestingly, recent research showed that sedentary behaviour (activities requiring low levels of energy expenditure, e.g., sitting and lying), independent of performance of physical activity, is strongly related to negative health outcomes and mortality $[5,6]$. Moreover, sedentary behaviour is associated with lower cognitive performance [7], which stresses the relevance of understanding the prevalence and characteristics of sedentary behaviour in the context of dementia. Therefore, we aim to objectively determine physical activity and sedentary behaviour characteristics of community-dwelling dementia patients compared to cognitively healthy age-, sex- and weight-matched controls. Secondary, we will explore whether increasing age attenuates physical activity and sedentary behaviour in dementia.

\section{Materials and Methods}

\section{Participants and Design}

In this cross-sectional study, persons with a dementia diagnosis aged $>60$ years who were ambulatory and community dwelling were included. Dementia diagnosis was based on comprehensive clinical assessment by a physician, typically including neuropsychological assessment and imaging. We used the Mini-Mental State Examination (MMSE) to indicate severity of cognitive impairment [8]. Baseline measurements of a longitudinal trial examining the effects of exercise on cognitive functioning in dementia were used for the current study [9]. Cognitively healthy controls were age, sex and weight matched to dementia patients and had no history of cognitive impairment (MMSE $>24$ [8]). All participants were ambulatory and community dwelling. The study protocol was approved by the local Medical Ethics Committee in accordance with the latest revision of the Declaration of Helsinki. Written informed consent was obtained from all participants.

\section{Physical Activity Monitoring}

Directly after screening, physical activity and sedentary behaviour were assessed by the Philips Actiwatch 2, a wrist-worn accelerometer validated in middle-aged females [10]. The Philips Actiwatch 2 contains an acceleration-responsive piezoelectric sensor which measures wrist accelerations in 3 directions every $30 \mathrm{~s}$. These wrist accelerations were translated into a number of counts that were used to estimate physical activity and sedentary behaviour. The accelerometer was worn for 7 days on the non-dominant wrist to provide a reliable estimate of physical activity and sedentary behaviour [11,12]. All participants wore the Actiwatch $24 \mathrm{~h}$ per day. The accelerometer was waterproof, and participants did not take if off during swimming or taking a shower. Therefore, non-wear time was not expected. Sleep intervals, including daytime naps, were filled in by the participants or their caregivers in a sleep diary.

Data Analysis

Data was uploaded using the Philips Actiware 6 software. Data from the first day of testing were excluded from analysis to give participants the opportunity to familiarize with the device. Sleep intervals 
Hartman et al.: Dementia Patients Are More Sedentary and Less Physically Active than Age- and Sex-Matched Cognitively Healthy Older Adults

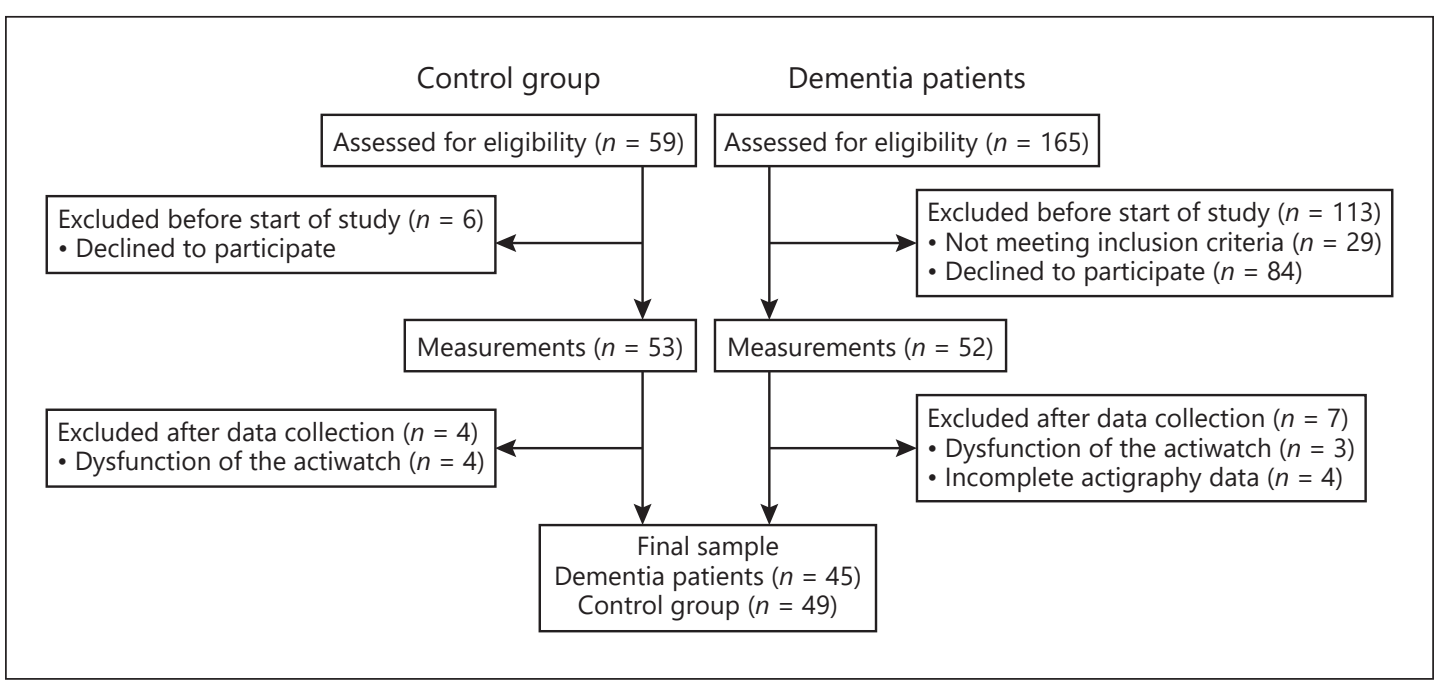

Fig. 1. Flowchart of participants.

were manually set by the researcher using the Philips Actiware 6 software [13] and excluded by custom software written in MATLAB R2014b (MathWorks, USA). Hereby, only the data of the waking hours remained. Participants were included if they provided at least 6 valid days ( $>10 \mathrm{~h}$ of waking data). Data was converted from counts per epoch into counts per minute (CPM). Cut-off points of 145, 145-274, 274-597 and $>597$ CPM were used for sedentary behaviour and very light, light-to-moderate and moderate-tovigorous physical activity, respectively [10]. To account for individual differences in waking time, our primary analysis expressed activity levels as a percentage of total (awake) measuring time. Interruptions in sedentary behaviour were defined as spending $1 \mathrm{~min}$ with $\geq 145 \mathrm{CPM}$ after $5 \mathrm{~min}$ with $<145 \mathrm{CPM}$. Prolonged sedentary behaviour was defined as spending 30 min with $<145$ CPM without 1 min of $>145$ CPM. Duration of average sedentary bout was defined by total time spent sedentary divided by the number of interruptions in sedentary behaviour.

\section{Statistical Analysis}

Statistical analyses were performed in IBM SPSS Statistics 20.0 (IBM SPSS; IBM Corp., Armonk, New York, NY, USA). We performed a complete case analysis, including only those participants that wore the Actiwatch for a minimum of 7 days. $\chi^{2}$ tests for categorical variables and independent samples $t$ tests for continuous variables were used to compare dementia patients and controls. Non-parametric tests were used for not normally distributed data (including physical activity and sedentary behaviour). To evaluate the impact of age, the same analyses were performed between participants aged $<80$ and $\geq 80$ years. All data are presented as median (interquartile range) unless stated otherwise. Level of significance was set at $p<0.05$.

\section{Results}

Forty-five dementia patients (mean \pm SD age $79.6 \pm 5.9$ years, MMSE $22.8 \pm 3.2$ ) and 49 controls (mean \pm SD age $80.0 \pm 7.7$ years, MMSE $29.0 \pm 1.2$ ) were included (Fig. 1). Sex, body mass index, walking aid use and number of comorbidities did not differ between groups (Table 1). MMSE was significantly lower in dementia patients $(p<0.001)$, and dementia patients received significantly more home care $(p<0.001)$. The majority of dementia patients and controls lived independently (93 and 98\%, respectively).

Total waking time tended to be lower in dementia patients than in controls (mean \pm SD $14.9 \pm 1.3$ vs. $15.4 \pm 1.0 \mathrm{~h} /$ day, $p=0.053$; Table 2 ). Dementia patients had significantly lower activity counts and spent more hours in categories reflecting lower-intensity activity (Table 


\begin{tabular}{l|l}
\hline DOI: 10.1159/000491995 & $\begin{array}{l}\text { (c) } 2018 \text { The Author(s). Published by S. Karger AG, Basel } \\
\text { www.karger.com/dem }\end{array}$
\end{tabular}

Hartman et al:: Dementia Patients Are More Sedentary and Less Physically Active than Age- and Sex-Matched Cognitively Healthy Older Adults

Table 1. Baseline characteristics

\begin{tabular}{|c|c|c|c|}
\hline & Control $(n=49)$ & Dementia $(n=45)$ & $p$ value \\
\hline Age, years & $80.0 \pm 7.7$ & $79.6 \pm 5.9$ & $0.744^{*}$ \\
\hline Females & $25(51.0)$ & $22(48.9)$ & $0.836^{\dagger}$ \\
\hline Body mass index & $25.5(4.0)$ & $26.3(5.0)$ & $0.411^{*}$ \\
\hline Mini-Mental State Examination ${ }^{\mathrm{a}}$ & $29.0 \pm 1.2$ & $22.8 \pm 3.2$ & $<0.001^{*}$ \\
\hline Walking aid users & $10(20.4)$ & $16(35.6)$ & $0.101^{\dagger}$ \\
\hline Home care receivers & $6(12.2)$ & $24(53.3)$ & $<0.001^{\dagger}$ \\
\hline Comorbidities $^{\mathrm{b}}$ & $2.7 \pm 1.8$ & $3.3 \pm 1.9$ & $0.150^{*}$ \\
\hline Residence & & & $0.629^{\dagger}$ \\
\hline Independent, alone & $18(36.7)$ & $14(31.1)$ & \\
\hline Independent, together & $30(61.2)$ & $28(62.2)$ & \\
\hline Care home & $1(2.0)$ & $3(6.7)$ & \\
\hline Nursing home & 0 & 0 & \\
\hline \multicolumn{4}{|l|}{ Dementia type } \\
\hline Alzheimer disease & $\mathrm{n} / \mathrm{a}$ & $25(55.6)$ & \\
\hline Vascular dementia & $\mathrm{n} / \mathrm{a}$ & $2(4.4)$ & \\
\hline Alzheimer disease/vascular dementia & $\mathrm{n} / \mathrm{a}$ & $12(26.7)$ & \\
\hline Dementia type not specified & $\mathrm{n} / \mathrm{a}$ & $6(13.3)$ & \\
\hline
\end{tabular}

Values are presented as means \pm standard deviations or $n(\%)$. Italics indicate statistical significance. $\mathrm{n} / \mathrm{a}$, not applicable. ${ }^{a}$ Scores on the Mini-Mental State Examination range from 0 (severe impairment) to 30 (no impairment). ${ }^{\mathrm{b}}$ Comorbidities are scored using the Older Persons and Informal Caregivers Survey-Minimum Dataset (TOPICS-MDS) with a theoretical range of $0-17$, and a higher score indicates more comorbidities [34]. * Differences between groups were tested with the independent samples $t$ test. ${ }^{\dagger}$ Differences between groups were tested with the $\chi^{2}$ test.

Table 2. Physical activity and sedentary behavior characteristics

\begin{tabular}{|c|c|c|c|}
\hline & Control $(n=49)$ & Dementia $(n=45)$ & $p$ value \\
\hline Total waking time, h/day & $15.4 \pm 1.0$ & $14.9 \pm 1.3$ & $0.053^{\dagger}$ \\
\hline Counts per minute, day ${ }^{-1}$ & $226 \pm 61$ & $186 \pm 76$ & $0.005^{\dagger}$ \\
\hline \multicolumn{4}{|l|}{ Absolute values, h/day } \\
\hline Sedentary time & $8.1(7.2-9.2)$ & $8.5(7.2-10.0)$ & 0.216 \\
\hline Very light intensity activity & $2.2(1.9-2.6)$ & $2.3(1.7-2.9)$ & 0.748 \\
\hline Light-to-moderate intensity activity & $3.5(2.7-4.0)$ & $2.7(2.0-3.7)$ & 0.006 \\
\hline Moderate-to-vigorous intensity activity & $1.5(0.8-2.0)$ & $0.8(0.4-1.5)$ & 0.001 \\
\hline \multicolumn{4}{|l|}{ Relative values, $\%$ of total measuring time } \\
\hline Sedentary time & $55(47-59)$ & $57(49-68)$ & 0.042 \\
\hline Very light intensity activity & $15(12-16)$ & $16(12-19)$ & 0.284 \\
\hline Light-to-moderate intensity activity & $22(18-25)$ & $20(15-23)$ & 0.017 \\
\hline Moderate-to-vigorous intensity activity & $10(5-13)$ & $5(2-10)$ & 0.001 \\
\hline \multicolumn{4}{|l|}{ Sedentary behavior characteristics } \\
\hline \multicolumn{4}{|l|}{ Number of interruptions in sedentary } \\
\hline behavior, day ${ }^{-1}$ & $28.2(26.2-32.5)$ & $27.2(24.5-31.0)$ & 0.195 \\
\hline \multicolumn{4}{|l|}{ Number of 30-min prolonged sedentary } \\
\hline bouts, day-1 & $2.0(0.9-3.3)$ & $2.3(1.0-4.1)$ & 0.227 \\
\hline Duration of average sedentary bout, min & $16.6(15.3-18.4)$ & $18.3(16.4-21.1)$ & 0.008 \\
\hline
\end{tabular}

Values are presented as means \pm standard deviations or medians (interquartile ranges). $p$ values represent Mann-Whitney $U$ test. Italics indicate statistical significance. ${ }^{\dagger}$ Differences between groups were tested with the independent samples $t$ test. 
Hartman et al.: Dementia Patients Are More Sedentary and Less Physically Active than

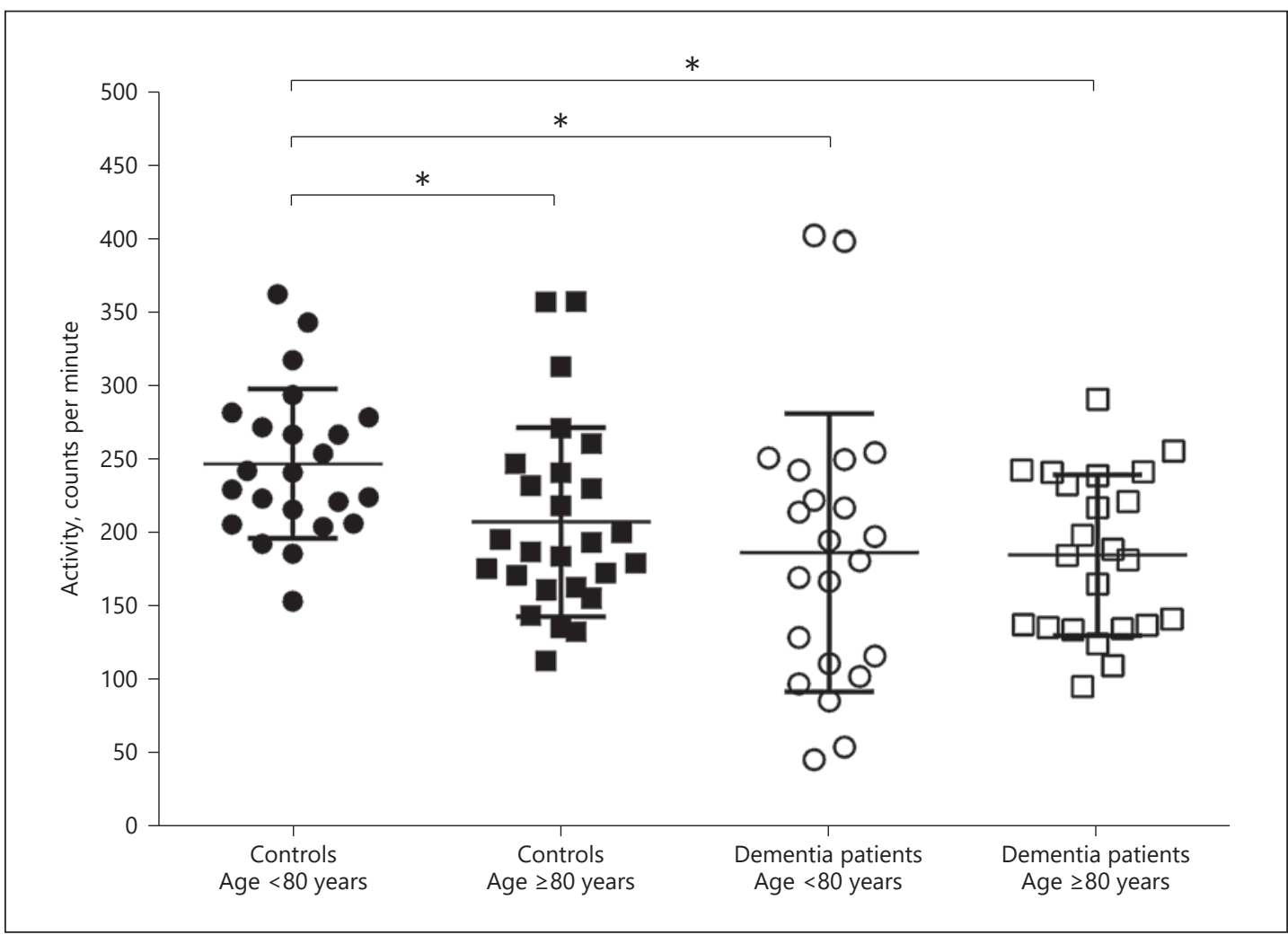

Fig. 2. Activity levels of dementia patients and cognitively healthy controls. Activity levels of younger and older age groups. Mean activity score (counts per minute) grouped by age. Controls age <80 years $(n=23)$, controls age $\geq 80$ years $(n=26)$, dementia patients age $<80$ years $(n=22)$, dementia patients age $\geq 80$ years $(n=23)$. Values represent medians and interquartile ranges. * Significant difference between: controls age $<80$ years and controls age $\geq 80$ years, $p=0.044$; controls age $<80$ years and dementia patients age $<80$ years, $p=0.004$; and controls age $<80$ years and dementia patients age $\geq 80$ years, $p=0.002$.

2). Relative sedentary time was significantly higher in dementia patients than in controls (57\% [49-68] vs. 55\% [47-59], $p=0.042$; Table 2). In addition, dementia patients spent a lower percentage of their waking time in light-to-moderate and moderate-to-vigorous intensity physical activity (20\% [15-23] vs. $22 \%$ [18-25] and 5\% [2-10] vs. $10 \%$ [5-13], $p=$ 0.017 and $p=0.001$, respectively). Number of interruptions in sedentary behaviour and prolonged sedentary bouts did not differ. The duration of sedentary bouts was significantly longer in dementia patients than in controls (18.3 [16.4-21.1] min vs. 16.6 [15.3-18.4] min, $p=0.008$; Table 2).

When comparing younger with older subgroups, older participants showed more walking aid use. Whilst older controls showed significantly less physical activity and more sedentary behaviour than their younger peers, no such differences were present between dementia patients aged $\geq 80$ years and those aged $<80$ years (Fig. 2). More specifically, younger controls had significantly higher activity counts, lower relative sedentary time, and spent more hours in categories reflecting light-to-moderate and moderate-to-vigorous intensity activity compared to older controls. Since no such differences were present between young and older dementia patients, differences in physical activity and sedentary behaviour characteristics between populations were most prominent in the younger groups (Fig. 2). 
Hartman et al.: Dementia Patients Are More Sedentary and Less Physically Active than Age- and Sex-Matched Cognitively Healthy Older Adults

\section{Discussion}

The aim of our study was to objectively investigate physical activity and sedentary behaviour characteristics of dementia patients compared to controls and assess whether age affects this comparison. First, in our relatively large sample, we found that dementia patients spent significantly more of their waking hours in a sedentary state and significantly less time in light-to-moderate and moderate-to-vigorous intensity activities. This may have clinically important consequences, given the observation of previous prospective studies that sedentary behaviour independently predicts all-cause mortality and morbidity [5, 14]. Secondly, we found that older age was associated with a decline in physical activity and increase in sedentary behaviour in controls, whilst no such age-related changes were found in dementia patients. Consequently, negligible differences in physical activity and sedentary behaviour characteristics were present when comparing older dementia patients and controls.

In line with observations from previous work $[15,16]$, our data confirm that communitydwelling dementia patients spend a large amount of time in sedentary behaviour and have low levels of physical activity. We add the novel finding that differences between dementia patients and controls remain when corrected for sleep time. Even though we used an accelerometer validated to measure physical activity and sedentary behaviour [10], time spent in moderate-to-vigorous intensity activity seems unusually high in both groups [17]. We have compared our findings to other studies that assessed physical activity and sedentary behaviour in older adults with and without dementia, and we note that sedentary behaviour ranged from 6.7 to $10.7 \mathrm{~h}$ between studies $[15,18,19]$. Moreover, the percentage of elderly meeting physical activity guidelines (150 $\mathrm{min} /$ week of moderate-to-vigorous physical activity) ranged from 27 to $69 \%$ [17, 20]. Differences in sedentary behaviour and physical activity duration between studies might relate to the use of different types of accelerometers, given that the reported studies used hip-worn accelerometers, wrist-worn accelerometers or questionnaires to estimate physical activity and sedentary behaviour [21]. However, this does not invalidate our primary comparison between subjects with dementia and healthy peers. Moreover, this highlights the importance of including a control group to provide valid interpretation of the results.

Our observation raises the question if differences in physical activity and sedentary behaviour are simply a consequence of dementia. A decline in executive functioning (i.e., necessary for goal-directed behaviour, such as physical activity) could lead to apathy [22], which is known to lower activity levels in Alzheimer disease patients [23]. However, it is important to realize that lower physical activity and higher sedentary behaviour have already been reported in the preclinical stages of dementia [24] and in subjects with mild cognitive impairment [25]. This might suggest that differences in physical activity and sedentary behaviour are causally linked to progression from mild cognitive impairment to later stages of dementia. Future research is necessary to answer this question on cause or effect. Nevertheless, since higher physical activity and lower sedentary behaviour are associated with better cognitive performance [7,25], benefits of interventions promoting physical activity and reducing sedentary behaviour should be investigated.

In response to our second research question, we found that older age was associated with a decline in physical activity and increase in sedentary behaviour in controls. This observation can partly be explained by deterioration of walking and mobility and increased disability with older age [26]. Interestingly, no further decline in physical activity and increase in sedentary behaviour was found in dementia patients, despite the age-related increased number of walking aid users. This striking result suggests that cognitive impairment in dementia has a great impact on physical activity and sedentary behaviour and may be more important than the impact of other factors, such as deterioration of walking and mobility. An 
Dementia

Cognitive Disorders

\begin{tabular}{l|l}
\hline Dement Geriatr Cogn Disord 2018:46:81-89 \\
\hline DOI: 10.1159/000491995 & $\begin{array}{l}\text { (c) 2018 The Author(s). Published by S. Karger AG, Basel } \\
\text { www.karger.com/dem }\end{array}$ \\
\hline
\end{tabular}

Hartman et al.: Dementia Patients Are More Sedentary and Less Physically Active than Age- and Sex-Matched Cognitively Healthy Older Adults

alternative explanation is that a (near) minimum level is achieved in the decline in levels of physical activity and sedentary behaviour in community-dwelling subjects [17].

In addition to the duration of sedentary behaviour, previous work revealed that the frequency of breaking up sitting (and, therefore, duration of each sedentary bout) may have clinical relevance. Breaks in sedentary behaviour can prevent cardiovascular impairments [5] and play a role in maintaining glycaemic control, which may positively influence brain health [27]. Our study found that the average duration of a sedentary bout was higher in dementia patients compared to controls. This observation suggests that not only reducing sedentary time, but also preventing prolonged sedentary bouts by regularly breaking up sedentary behaviour, can be targeted as a lifestyle intervention [28].

\section{Limitations}

Since we only included community-dwelling patients, our results cannot be generalized to institutionalized dementia patients. Furthermore, dementia patients in our study were enrolled in an exercise trial [9]. Therefore, this group may be more motivated to be physically active. Nonetheless, significant differences in physical activity characteristics were observed. Furthermore, physical activity characteristics were measured by wrist-worn accelerometry, which is associated with limited discriminative capacity between sedentary and very-light intensity activities [29]. Consequently, differences between dementia patients and controls might even be more pronounced. Another limitation relates to our accelerometer, which has only been validated in a group of middle-aged females [10] and was unable to correct for potential presence of short ( 1-min) periods of non-wear time. Nonetheless, we do not expect this will invalidate our findings of between-group differences in physical activity and sedentary behaviour. In addition, using wrist-worn accelerometry may explain the 2 outliers in the younger dementia patients (Fig. 2), which could relate to restless arm movements. Whilst this may affect exploring individual differences in physical activity versus sedentary behaviour characteristics, significant differences remained present at group level. Furthermore, we did not discriminate between types of sedentary activities. Cognitively challenging sedentary activities, such as reading, might have a protective effect on cognition and are, therefore, less harmful than passive sedentary activities (e.g., television viewing) [30]. The final limitation is use of the MMSE as a cognitive screening instrument since this measure, especially in healthy highly educated older adults, has limited discriminative power to detect mild cognitive deficits $[31,32]$. It is important to indicate that diagnosis of dementia was not made using the MMSE but included standard clinical procedures (including imaging if required).

\section{Clinical Relevance}

Knowledge about physical activity characteristics across the entire activity spectrum in dementia is highly relevant given that physical activity is an important factor accelerating development and progression of dementia [3, 7]. In addition, a high amount of sedentary behaviour and low amount of physical activity are associated with higher mortality and morbidity [5, 6]. Even though we found relatively small differences in physical activity characteristics between dementia patients and controls, these may be highly relevant. For example, even very short breaks of light intensity activity (i.e., 2 min of walking) can already prevent acute metabolic [33] and cardiovascular [5] impairments. This underlines the importance to develop interventions suitable for this vulnerable patient group to safely engage in light intensity activities. However, future research should first explore the role of physical activity and sedentary behaviour in the progression and prevention of dementia. 


\section{Conclusion}

In the current study, we objectively demonstrated that dementia patients spend significantly more of their waking hours in sedentary behaviour and spend less time in light-tomoderate and moderate-to-vigorous intensity physical activity. Moreover, we found that older age attenuated sedentary behaviour and physical activity in controls, whilst this agerelated decline was absent in dementia patients. This means that patients with dementia (independent of age) lead a physically inactive lifestyle characterized by significant time spent sedentary.

Taken together, these data improve our understanding of physical activity and sedentary behaviour characteristics in this highly relevant patient group and imply that targeting sedentary behaviour and physical activity may be relevant in dementia patients, especially at a younger age.

\section{Disclosure Statement}

There are no conflicts of interest.

\section{Funding Sources}

This research did not receive any specific grant from funding agencies in the public, commercial or notfor-profit sectors.

\section{References}

1 Perera G, Pedersen L, Ansel D, Alexander M, Arrighi HM, Avillach P, et al: Dementia prevalence and incidence in a federation of European Electronic Health Record databases - The European Medical Informatics Framework resource. Alzheimers Dement 2018;14:130-139.

2 Kavirajan H, Schneider LS: Efficacy and adverse effects of cholinesterase inhibitors and memantine in vascular dementia: a meta-analysis of randomised controlled trials. Lancet Neurol 2007;6:782-792.

3 Ashby-Mitchell K, Burns R, Shaw J, Anstey KJ: Proportion of dementia in Australia explained by common modifiable risk factors. Alzheimers Res Ther 2017;9:11.

4 Groot C, Hooghiemstra A, Raijmakers P, Van Berckel B, Scheltens P, Scherder E, et al: The effect of physical activity on cognitive function in patients with dementia: a meta-analysis of randomized control trials. Ageing Res Rev 2016;25:13-23.

5 Carter S, Hartman Y, Holder S, Thijssen DH, Hopkins ND: Sedentary behavior and cardiovascular disease risk: mediating mechanisms. Exerc Sport Sci Rev 2017;45:80-86.

6 Ekelund U, Steene-Johannessen J, Brown WJ, Fagerland MW, Owen N, Powell KE, et al: Does physical activity attenuate, or even eliminate, the detrimental association of sitting time with mortality? A harmonised metaanalysis of data from more than 1 million men and women. Lancet 2016;388:1302-1310.

7 Falck RS, Davis JC, Liu-Ambrose T: What is the association between sedentary behaviour and cognitive function? A systematic review. Br J Sports Med 2017;51:800-811.

8 Folstein MF, Folstein SE, McHugh PR: "Mini-mental state." A practical method for grading the cognitive state of patients for the clinician. J Psychiatr Res 1975;12:189-198.

9 Karssemeijer EGA, Bossers WJR, Aaronson JA, Kessels RPC, Olde Rikkert MGM: The effect of an interactive cycling training on cognitive functioning in older adults with mild dementia: study protocol for a randomized controlled trial. BMC Geriatr 2017;17:73.

10 Neil-Sztramko SE, Rafn BS, Gotay CC, Campbell KL: Determining activity count cut-points for measurement of physical activity using the Actiwatch 2 accelerometer. Physiol Behav 2017;173:95-100.

11 Hart TL, Swartz AM, Cashin SE, Strath SJ: How many days of monitoring predict physical activity and sedentary behaviour in older adults? Int J Behav Nutr Phys Act 2011;8:62.

12 Sasaki JE, Junior JH, Meneguci J, Tribess S, Marocolo Junior M, Stabelini Neto A, et al: Number of days required for reliably estimating physical activity and sedentary behaviour from accelerometer data in older adults. J Sports Sci 2018;36:1572-1577. 
Hartman et al.: Dementia Patients Are More Sedentary and Less Physically Active than Age- and Sex-Matched Cognitively Healthy Older Adults

13 Ancoli-Israel S, Cole R, Alessi C, Chambers M, Moorcroft W, Pollak CP: The role of actigraphy in the study of sleep and circadian rhythms. Sleep 2003;26:342-392.

14 van der Ploeg HP, Chey T, Korda RJ, Banks E, Bauman A: Sitting time and all-cause mortality risk in 222497 Australian adults. Arch Intern Med 2012;172:494-500.

15 van Alphen HJ, Volkers KM, Blankevoort CG, Scherder EJ, Hortobagyi T, van Heuvelen MJ: Older adults with dementia are sedentary for most of the day. PLoS One 2016;11:e0152457.

16 James BD, Boyle PA, Bennett DA, Buchman AS: Total daily activity measured with actigraphy and motor function in community-dwelling older persons with and without dementia. Alzheimer Dis Assoc Disord 2012; 26:238-245.

17 Keadle SK, McKinnon R, Graubard BI, Troiano RP: Prevalence and trends in physical activity among older adults in the United States: a comparison across three national surveys. Prev Med 2016;89:37-43.

18 Harvey JA, Chastin SF, Skelton DA: How sedentary are older people? A systematic review of the amount of sedentary behavior. J Aging Phys Act 2015;23:471-487.

19 Manns P, Ezeugwu V, Armijo-Olivo S, Vallance J, Healy GN: Accelerometer-derived pattern of sedentary and physical activity time in persons with mobility disability: National Health and Nutrition Examination Survey 2003 to 2006. J Am Geriatr Soc 2015;63:1314-1323.

20 Visser M, Wijnhoven HAH, Comijs HC, Thomese F, Twisk JWR, Deeg DJH: A healthy lifestyle in old age and prospective change in four domains of functioning. J Aging Health 2018:898264318774430.

21 Falck RS, McDonald SM, Beets MW, Brazendale K, Liu-Ambrose T: Measurement of physical activity in older adult interventions: a systematic review. Br J Sports Med 2016;50:464-470.

22 McPherson S, Fairbanks L, Tiken S, CummingsJL, Back-Madruga C: Apathy and executive function in Alzheimer's disease. J Int Neuropsychol Soc 2002;8:373-381.

23 David R, Mulin E, Friedman L, Le Duff F, Cygankiewicz E, Deschaux O, et al: Decreased daytime motor activity associated with apathy in Alzheimer disease: an actigraphic study. Am J Geriatr Psychiatry 2012;20:806-814.

24 Sabia S, Dugravot A, Dartigues JF, Abell J, Elbaz A, Kivimaki M, et al: Physical activity, cognitive decline, and risk of dementia: 28 year follow-up of Whitehall II cohort study. BMJ 2017;357:j2709.

25 Falck RS, Landry GJ, Best JR, Davis JC, Chiu BK, Liu-Ambrose T: Cross-sectional relationships of physical activity and sedentary behavior with cognitive function in older adults with probable mild cognitive impairment. Phys Ther 2017; 97:975-984.

26 Koolhaas CM, van Rooij FJA, Schoufour JD, Cepeda M, Tiemeier H, Brage S, et al: Objective measures of activity in the elderly: distribution and associations with demographic and health factors. J Am Med Dir Assoc 2017; 18:838-847.

27 Wheeler MJ, Dempsey PC, Grace MS, Ellis KA, Gardiner PA, Green DJ, et al: Sedentary behavior as a risk factor for cognitive decline? A focus on the influence of glycemic control in brain health. Alzheimers Dement (NY) 2017;3:291-300.

28 Dempsey PC, Owen N, Biddle SJH, Dunstan DW: Managing sedentary behavior to reduce the risk of diabetes and cardiovascular disease. Curr Diab Rep 2014;14:522.

29 Tudor-Locke C, Barreira TV, Schuna JM: Comparison of step outputs for waist and wrist accelerometer attachment sites. Med Sci Sports Exerc 2015;47:839-842.

30 Kikuchi H, Inoue S, Sugiyama T, Owen N, Oka K, Nakaya T, et al: Distinct associations of different sedentary behaviors with health-related attributes among older adults. Prev Med 2014;67:335-339.

31 Tangalos EG, Smith GE, Ivnik RJ, Petersen RC, Kokmen E, Kurland LT, et al: The Mini-Mental State Examination in general medical practice: clinical utility and acceptance. Mayo Clin Proc 1996;7:829-837.

32 O’Bryant SE, Humphreys JD, Smith GE, Ivnik RJ, Graff-Radford NR, Petersen RC, et al: Detecting dementia with the Mini-Mental State Examination in highly educated individuals. Arch Neurol 2008;65:963-967.

33 Dunstan DW, Kingwell BA, Larsen R, Healy GN, Cerin E, Hamilton MT, et al: Breaking up prolonged sitting reduces postprandial glucose and insulin responses. Diabetes Care 2012;35:976-983.

34 Lutomski JE, Baars MA, Schalk BW, Boter H, Buurman BM, den Elzen WP, et al: The development of the Older Persons and Informal Caregivers Survey Minimum DataSet (TOPICS-MDS): a large-scale data sharing initiative. PLoS One 2013;8:e81673. 